\title{
Anomalous thermodynamics of Coulomb interacting massless Dirac fermions in two spatial dimensions
}

\author{
Oskar Vafek ${ }^{1}$ \\ ${ }^{1}$ National High Magnetic Field Laboratory and Department of Physics, \\ Florida State University, Tallahassee, Florida 32306, USA
}

(Dated: April 29, 2019)

\begin{abstract}
It is argued that the specific heat of $N$ massless Dirac fermions in 2 spatial dimensions interacting with $1 / \mathrm{r}$ Coulomb interactions is suppressed logarithmically relative to its non-interacting counterpart. The (dimensionless) coefficient of the logarithm is calculated in a closed form in the leading order in large $N$ expansion, but to all orders in the effective fine structure constant, $\alpha_{F}$, a procedure which takes into account finite temperature screening. This effect is expected to occur in a single layer graphene embedded in a dielectric medium. Its dependence on the dielectric constant is calculated analytically.
\end{abstract}

Understanding properties of quantum matter confined to two spatial dimensions has been at the forefront of theoretical physics [1]. The lowered dimensionality is believed to increase the role of interactions and, together with quantum statistics, lead to anomalies in various physical observables [2, 3,, 4$]$.

In the case of Fermi-Dirac systems, the role of (short range) interactions has been studied in the pioneering work of Landau [5]. What is now understood as the Fermi liquid paradigm explains the low temperature properties of the degenerate Fermi-Dirac systems as arising from a dilute gas of weakly interacting quasiparticles whose quantum numbers are the same as those of noninteracting fermions. In this context, it is also well known that a degenerate assembly of electrons with a rotationally invariant Fermi surface, in either three or two spatial dimensions, interacting with $1 / r$ Coulomb interactions, can be described by the Fermi liquid theory [6]. Ultimately, this is due to the ability of the mobile carriers to screen the long ranged interaction beyond the distances of the order of the inter-electron separation. One of the most direct observable consequences for a metal is the linear temperature dependence of the specific heat at asymptotically low temperatures i.e. $\lim _{T \rightarrow 0} c_{V} / T=\gamma$ [7, 8].

The situation is less clear in the case of a semimetal, which can be thought of loosely as the Fermi surface shrunk to a point. More precisely, in this case, a conduction band and a valence band typically touch at a discrete set of points with dispersion which vanishes linearly with the wavenumber near each such point, being in a sense a critical point between a metal and an insulator. If the chemical potential lies at such a point of degeneracy, the absence of the Fermi surface implies that, unlike in a metal, the long ranged interactions are screened only by thermally excited carriers, with the screening length of the order of the thermal length, $\hbar v_{F} / k_{B} T$. For a typical Fermi velocity $\sim 10^{6} \mathrm{~m} / \mathrm{s}$, even at room temperature the screening length can therefore be much longer than the interatomic spacing. The effect of the Coulomb in- teractions on 3D semimetals was investigated in Ref. 9] where it was argued that, unlike in a metal, the Coulomb interaction causes the energy spectrum to differ from a purely linear spectrum by a logarithmic factor.

In this work, I study the effects of the Coulomb interactions on the thermodynamic properties of massless Dirac fermions in 2D, which can be used as a low energy description of a single-layer graphene, and find that the poorly screened electron-electron interaction leads to anomalies in the quasiparticle thermodynamics.

In the absence of interactions, the free energy density is (up to a temperature independent constant)

$$
f_{0}=-N k_{B} T^{3} \frac{3 \zeta(3)}{4 \pi v_{F}^{2}}
$$

where $N$ is the number of the 2 component Dirac flavors ( $N=4$ in the single layer graphene) and $\zeta(n)$ is the Riemann zeta function. The strength of the electronelectron interaction is given by the dimensionless coupling constant $\alpha_{F}=e^{2} /\left(\epsilon \hbar v_{F}\right)$, which is the effective fine structure constant, where $\epsilon$ describes the polarizability of the surrounding medium. If $\epsilon$ is large, then $\alpha_{F}$ is small, and to first order in $\alpha_{F}, v_{F}$ receives a logarithmic correction $\frac{1}{4} v_{F} \alpha_{F} \ln \left[\frac{\Lambda}{k}\right]$, where $\Lambda$ is a short wavelength cuttoff. Such logarithmic enhancement of the otherwise linear dispersion near the Dirac point, would imply logarithmic suppression of the specific heat. It is not clear, however, whether such simpleminded reasoning suffices. Similar reasoning in the case of an electron gas with a Fermi surface would also lead to a logarithmic suppression of the effective mass, the result known to be incorrect due to screening. Therefore, the key issue in this context is the effect of the thermal screening on the specific heat in the 2D Dirac case.

To answer this question, I set up a large $N$ expansion while keeping $\alpha_{F} N$ fixed and arbitrary. To the leading order in large $N$, this is equivalent to a random phase approximation (RPA). I find that the free energy density 
receives a logarithmic correction

$$
\delta f=\frac{3 \zeta(3)}{\pi^{2}}\left[\frac{8}{\pi} \lambda^{2} g(\lambda)-\frac{2}{\lambda}\right] \frac{k_{B}^{3} T^{3}}{\hbar^{2} v_{F}^{2}} \ln \left(\frac{T_{U V}}{T}\right)
$$

where $\lambda=\alpha_{F} N \pi / 8$, the large temperature cutoff is related to the short wavelength cutoff by $\hbar v_{F} \Lambda=k_{B} T_{U V}$, and the function $g$ is given by the Eq.(12).

To the same order in the large $N$ expansion, the above result can be interpreted as a free energy density of $2 \mathrm{~N}$ modes [10] with dispersion

$$
\epsilon_{\eta}(k)=\hbar v_{F} k\left(1+\eta \ln \left[\frac{\Lambda}{k}+1\right]\right)
$$

where

$$
\eta=\frac{1}{N}\left[\frac{16}{\pi^{2}} \lambda^{2} g(\lambda)-\frac{4}{\pi \lambda}\right]
$$

I find that as $\lambda \rightarrow 0, \eta=2 \lambda /(\pi N)$ while for $\lambda \rightarrow \infty$, $\eta=8 /\left(\pi^{2} N\right)$ (see Fig 1). The expression (11) can be understood as the first non-trivial term in the Taylor expansion of

$$
f(T, \Lambda, \eta)=-2 N k_{B} T \int_{0}^{\infty} \frac{d k}{2 \pi} k \ln \left[1+\exp \left(-\frac{\epsilon_{\eta}(k)}{k_{B} T}\right)\right]
$$

Note that in the limit of $\lambda \rightarrow 0$, the above expression coincides with the free energy calculated within the Hartree-Fock approximation. As $T \rightarrow 0$, the free energy in (2) vanishes as $-T^{3} / \ln ^{2} T$ [11, 12]. Thus the suppression of the specific heat $c_{V}=-T \partial^{2} f / \partial T^{2}$ relative to the non-interacting case persists when the polarization effects are included.

The logarithmic divergence of the particle's group velocity at low wavenumbers may seem to violate causality. This is an artifact of the approximation which treats the $1 / r^{2}$ Coulomb forces as instantaneous. Were we to include the retardation effects introduced by coupling the fermions to a (quantum) 3D electromagnetic gauge field, the Fermi velocity would not grow without bound at low $k$, but instead saturate to the speed of light [13]. However, since the growth of $v_{F}$ is only logarithmic, the effects of retardation on the specific heat would be practically unobservable for $c \gg v_{F}$. For all practical purposes, the above non-relativistic expression will therefore span the physically relevant regime.

Below, I justify the above claims. For convenience, I work in units where $\hbar=k_{B}=v_{F}=1$ and restore the physical units in the final expressions. Then, the Hamiltonian is

$$
\mathcal{H}=\mathcal{H}_{0}+\hat{V}
$$

The free part of $\mathcal{H}$ is

$$
\mathcal{H}_{0}=\sum_{j=1}^{N} \int d^{2} \mathbf{r}\left[\psi_{j}^{\dagger}(\mathbf{r}) \mathbf{p} \cdot \sigma \psi_{j}(\mathbf{r})\right]
$$

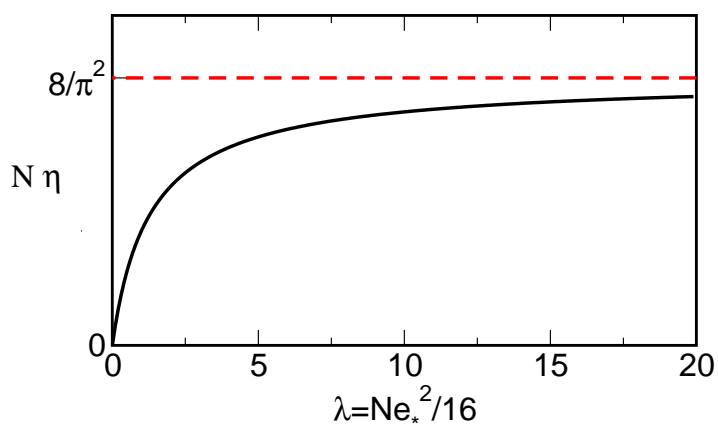

FIG. 1: The coefficient $\eta=\frac{1}{N}\left[\frac{16}{\pi^{2}} \lambda^{2} g(\lambda)-\frac{4}{\pi \lambda}\right]$ of the logarithmic increase of the free energy in Eq. (2) vs. $\lambda=$ $N e_{*}^{2} / 16=N \frac{\pi}{8} \frac{e^{2}}{\epsilon \hbar v_{F}}$. The closed form expression for $g$ is given in Eq.12). For small $\lambda, \eta=2 \lambda /(\pi N)$ while for $\lambda \rightarrow \infty$, $\eta=8 /\left(\pi^{2} N\right)$.

where $\mathbf{p}=-i \nabla, \sigma$ 's are the Pauli matrices, and $\psi_{j}(\mathbf{r})$ 's are two component anticommuting Fermi fields. $N$ is the number of the Dirac fermion flavors which, in the condensed matter setting, equals the number of valleys times the number of spin directions $(N=4$ for a singlelayer graphene). The interaction part of $\mathcal{H}$ comes from the (3D) Coulomb interaction between the charge density fluctuations and reads

$$
\hat{V}=\frac{1}{2} \int d^{2} \mathbf{r} d^{2} \mathbf{r}^{\prime}\left[\delta \hat{n}(\mathbf{r}) \frac{e^{2}}{\epsilon} \frac{1}{\left|\mathbf{r}-\mathbf{r}^{\prime}\right|} \delta \hat{n}\left(\mathbf{r}^{\prime}\right)\right]
$$

Here $\delta \hat{n}(\mathbf{r})=\sum_{j} \psi_{j}^{\dagger}(\mathbf{r}) \psi_{j}(\mathbf{r})$, and $\epsilon$ is dielectric constant of any potential surrounding insulator. Note that in our units $e^{2} / \epsilon$, which is the analog of a fine structure constant, is dimensionless.

Under a scale transformation $\mathbf{r} \rightarrow b \mathbf{r}$ the Hamiltonian (3) appears to transform as $\mathcal{H} \rightarrow b^{-1} \mathcal{H}$. This might suggest that even in the interacting theory, the temperature dependent part of the free energy density $f$ goes as $T^{3}$. However, since the theory is well defined in the UV only in the presence a short distance cutoff $\Lambda^{-1}$, this symmetry and the $T^{3}$ form will in general be violated.

In the large $N$ expansion the leading non-trivial correction to the free energy density due to interactions is

$$
\begin{aligned}
\delta f & =\int_{0}^{\Lambda} \frac{d q q}{2 \pi} \int_{0}^{\infty} \frac{d \Omega}{2 \pi} \operatorname{coth} \frac{\Omega}{2 T}\left\{\tan ^{-1}\left[\frac{\Im m \Pi_{0}^{r e t}(q, \Omega, T)}{\frac{q}{e_{*}^{2}}+\Re e \Pi_{0}^{r e t}(q, \Omega, T)}\right]\right. \\
& \left.-\tan ^{-1}\left[\frac{\Im m \Pi_{0}^{r e t}(q, \Omega, 0)}{\frac{q}{e_{*}^{2}}+\Re e \Pi_{0}^{r e t}(q, \Omega, 0)}\right]\right\}
\end{aligned}
$$

where $e_{*}^{2}=2 \pi e^{2} / \epsilon . \Pi_{0}^{r e t}(q, \omega, T)$ is the retarded polarization function of the free fermions. 
For $\Omega^{2}>\mathbf{q}^{2}$ :

$$
\begin{aligned}
& \Re e \Pi_{0}^{r e t}(q, \Omega, T)=N T \frac{\ln 2}{\pi}-\frac{N q^{2}}{4 \pi \sqrt{\Omega^{2}-q^{2}}} \int_{1}^{\infty} d x\left[\frac{\sqrt{x^{2}-1}}{1+\exp \left|\frac{|\Omega|-q x}{2 T}\right|}-\frac{\sqrt{x^{2}-1}}{1+\exp \left|\frac{|\Omega|+q x}{2 T}\right|}\right] \\
& \Im m \Pi_{0}^{r e t}(q, \Omega, T)=\operatorname{sgn} \Omega \frac{N q^{2}}{4 \pi \sqrt{\Omega^{2}-q^{2}}} \int_{-1}^{1} d x \sqrt{1-x^{2}}\left[\frac{1}{2}-\frac{1}{1+\exp \frac{|\Omega|+q x \mid}{2 T}}\right]
\end{aligned}
$$

while for $\mathbf{q}^{2}>\Omega^{2}$

$$
\begin{aligned}
& \Re e \Pi_{0}^{r e t}(q, \Omega, T)=N T \frac{\ln 2}{\pi}+\frac{N q^{2}}{4 \pi \sqrt{q^{2}-\Omega^{2}}} \int_{-1}^{1} d x \sqrt{1-x^{2}}\left[\frac{1}{2}-\frac{1}{1+\exp \left|\frac{q x+|\Omega|}{2 T}\right|}\right] \\
& \Im m \Pi_{0}^{r e t}(q, \Omega, T)=\operatorname{sgn} \Omega \frac{N q^{2}}{4 \pi \sqrt{q^{2}-\Omega^{2}}} \int_{1}^{\infty} d x\left[\frac{\sqrt{1-x^{2}}}{1+\exp \frac{q x-|\Omega|}{2 T}}-\frac{\sqrt{1-x^{2}}}{1+\exp \frac{q x+|\Omega|}{2 T}}\right]
\end{aligned}
$$

At $T=0, \Pi_{0}^{r e t}(q, \Omega, 0)=$

$$
\frac{N}{16} \frac{q^{2}}{\sqrt{\left|q^{2}-\Omega^{2}\right|}}\left\{\theta\left(q^{2}-\Omega^{2}\right)+i \operatorname{sgn} \Omega \theta\left(\Omega^{2}-q^{2}\right)\right\} .
$$

Note that the interaction correction to the free energy has the scaling form

$$
\delta f\left(T, N, e_{*}^{2}, \Lambda\right)=T^{3} \mathcal{F}\left(e_{*}^{2} N, \Lambda / T\right),
$$

and in the low temperature limit of interest $\frac{\Lambda}{T} \gg 1$. I now proceed with the evaluation of Eq.(66) in this limit.

Consider first the static regime $q^{2}>\Omega^{2}$. As can be readily seen from Eq.(10), for large $q \Im m \Pi_{0}^{r e t}(q, \Omega, T)$ is small compared to $q / e_{*}^{2}$ and the real part, and the latter can be approximated by $\Re e \Pi_{0}^{r e t}(q, \Omega, 0)$ to the same order. As a result, the contribution to $\delta f$ from the static regime is

$$
\begin{array}{r}
T^{3} \int^{\frac{\Lambda}{T}} \frac{d q q^{2}}{2 \pi} \frac{N e_{*}^{2}}{4 \pi} \int_{0}^{1} \frac{d y}{2 \pi} \frac{1}{\sqrt{1-y^{2}}+\frac{N e_{*}^{2}}{16}} \times \\
\int_{1}^{\infty} d x\left[\frac{\sqrt{x^{2}-1}}{1+\exp \frac{q(x-y)}{2}+1}-\frac{\sqrt{x^{2}-1}}{1+\exp \frac{q(x+y)}{2}+1}\right] \\
\rightarrow \frac{8 \zeta\left(\frac{5}{2}\right)}{\pi^{5 / 2}}\left(1-\frac{\sqrt{2}}{4}\right) \sqrt{\frac{\Lambda}{T}}-\frac{48 \zeta(3)}{\pi^{2}} \frac{1}{N e_{*}^{2}} \ln \frac{\Lambda}{2 T}
\end{array}
$$

where the last line represents only the most divergent contribution.

Similarly, the most singular contribution from the dynamic regime, $\Omega^{2}>q^{2}$, can be evaluated by expanding (6) to first order in deviation of $\Im m \Pi_{0}^{r e t}(q, \Omega, T)$ from $\Im m \Pi_{0}^{r e t}(q, \Omega, 0)$ as well as to the first order in $\Re e \Pi_{0}^{r e t}(q, \Omega, T)$, both of which vanish at large $q$. The algebra is somewhat tedious and I just state the final result for the asymptotic expansion in $\Lambda / T$ :

$$
\begin{aligned}
& -\frac{8 \zeta\left(\frac{5}{2}\right)}{\pi^{5 / 2}}\left(1-\frac{\sqrt{2}}{4}\right) \sqrt{\frac{\Lambda}{T}}-\frac{48 \zeta(3)}{\pi^{2}} \frac{1}{N e_{*}^{2}} \ln \frac{\Lambda}{2 T} \\
& +\frac{3 \zeta(3)}{32 \pi^{3}} N^{2} e_{*}^{4} \times g\left(\frac{N e_{*}^{2}}{16}\right) \times \ln \frac{\Lambda}{2 T}
\end{aligned}
$$

where the function $g(x)$ is

$$
g(x)=\int_{0}^{\infty} d y \frac{(1+y)^{2}}{\sqrt{y(2+y)}\left(y(2+y)+x^{2}\right)^{2}}
$$

This integral can be evaluated by a substitution followed by a partial fraction decomposition and one finds

$$
\begin{aligned}
& g(x)=\frac{1}{2 x^{2}}+\frac{\tan ^{-1}\left[\frac{\sqrt{1-x^{2}}}{x}\right]}{2 x^{3} \sqrt{1-x^{2}}} ; \quad 0<x<1 \\
& g(x)=\frac{1}{2 x^{2}}+\frac{1}{4 x^{3} \sqrt{x^{2}-1}} \ln \left[\frac{x+\sqrt{x^{2}-1}}{x-\sqrt{x^{2}-1}}\right] ; \quad x>1
\end{aligned}
$$

Combining everything together I find that up to $\mathcal{O}\left(\frac{1}{N}\right)$ :

$f=-N \frac{3 \zeta(3)}{4 \pi} T^{3}+\left[\frac{24 \zeta(3)}{\pi^{3}} \lambda^{2} g(\lambda)-\frac{6 \zeta(3)}{\pi^{2}} \frac{1}{\lambda}\right] T^{3} \ln \frac{\Lambda}{2 T}$

where $\lambda=N e_{*}^{2} / 16$. This is the result quoted in Eq.(1). The corresponding function $\eta=\frac{1}{N}\left[\frac{16}{\pi^{2}} \lambda^{2} g(\lambda)-\frac{4}{\pi \lambda}\right]$ is plotted in Fig. (11). Note that the contribution from small $q$, which also includes the effect of the thermoplasma mode [14], gives only terms of order $T^{3}$.

If we interpret the above expression as an expansion of the free energy given in Eq.(2), the low temperature specific heat is subquadratic. Fig. (2) shows this suppression for realistic material parameters pertinent to the single-layer graphene. 


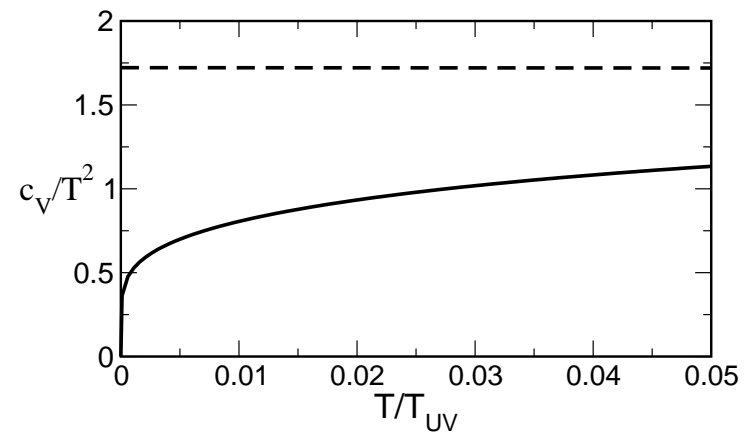

FIG. 2: The suppression of the specific heat (per Dirac cone) relative to the non-interacting result $c_{V}^{(0)}=\frac{9 \zeta(3)}{2 \pi} k_{B}^{3} T^{2} /\left(\hbar v_{F}\right)^{2}$ (dashed line) vs. $T / T_{U V}$. This plot is for $N=4, v_{F}=$ $10^{6} \mathrm{~m} / \mathrm{s}, \epsilon=1$ which gives $\eta \approx 0.14$.

It is interesting to make a connection between this result and a seemingly unrelated one of Ref.\#[15], where the authors studied the specific heat of $N$ massless 2D Dirac fermions coupled only to the space component of a 2D gauge field within the large $N$ expansion. Unlike our Eq.(11), the correction to the free energy found numerically in [15] has the opposite sign, i.e. the specific heat is enhanced relative to the non-interacting case. The numerical coefficient of the $T^{2} \ln \frac{1}{T}$ enhancement of $c_{V}$ is 2.79 [15], while in this work I find that upon setting $\lambda \rightarrow \infty$ the coefficient of the specific heat $T^{2} \ln T$ suppression is $72 \zeta(3) / \pi^{3} \approx 2.791$. The opposite signs of these terms can be understood as follows: setting $\lambda$ to infinity in Eq.\#(1), recovers the leading logarithmic contribution to the specific heat of $\mathrm{QED}_{3}$ within the large $N$ expansion arising from coupling solely to the time component of the gauge field. However, due to the Lorenz invariance of $\mathrm{QED}_{3}$, the leading logarithmic contributions from the time and the space components of the gauge field must cancel exactly [16]. It is in this sense that the result in Eq.(11) is consistent with Ref. 15].

In conclusion, I found that the thermodynamic signature of the long range electron-electron interactions in a two dimensional semimetal are much more pronounced than in the case of a metal. This stems from the in- ability of the charge carriers to screen the long range interactions, and as a result the density of states is effectively suppressed near the Dirac point. The experimental observation of the effect would be an important step towards our understanding of critical massless matter.

I wish to thank Professor Tesanovic for useful discussions.

[1] S. Kivelson, D.-H. Lee, and S.-C. Zhang, Scientific American 274, 64 (1996).

[2] D. A. Bonn, Nature Physics 2, 159 (2006).

[3] E. Abrahams, S. V. Kravchenko, and M. P. Sarachik, Rev. Mod. Phys. 73, 251 (2001).

[4] A. Casey, H. Patel, J. Nyéki, B. P. Cowan, and J. Saunders, Phys. Rev. Lett. 90, 115301 (2003).

[5] L. Landau, Soviet Physics JETP 3, 920 (1957).

[6] D. Pines and P. Nozieres, The Theory of Quantum Liquids (Perseus Books, Reading, Massachusetts, 1988).

[7] M. Gell-Mann, Phys. Rev. 106, 369 (1957).

[8] However, unlike in a free electron gas, the temperature corrections are non-analytic in $T^{2}$; for theory and experiment see for instance [17] and [18] respectively.

[9] A. A. Abrikosov and S. D. Beneslavskii, Sov. Phys. JETP 32, 699 (1971).

[10] Not counting spin, there are two modes associated with each Dirac cone.

[11] Showing that this dispersion indeed leads to $f \sim$ $-T^{3} / \ln ^{2} T$ as $T \rightarrow 0$ is facilitated by using the (secondary branch of the) Lambert $\mathrm{W}$ function and its asymptotic expansion, c.f. [12].

[12] R. M. Corless, G. H. Gonnet, D. E. G. Hare, D. J. Jeffrey, and D. E. Knuth, Adv. in Comp. Math. 5, 329 (1996).

[13] J. Gonzales, F. Guinea, and M. A. H. Vozmediano, Nucl. Phys. B 424, 595 (1994).

[14] O. Vafek, Phys. Rev. Lett. 97, 266406 (2006).

[15] D. H. Kim, P. A. Lee, and X.-G. Wen, Phys. Rev. Lett. 79, 2109 (1997).

[16] O. Vafek and Z. Tešanović, Phys. Rev. Lett. 91, 237001 (2003).

[17] A. V. Chubukov, D. L. Maslov, and A. J. Millis, Phys. Rev. B 73, 045128 (2006).

[18] D. S. Greywall, Phys. Rev. B 27, 2747 (1983). 